\title{
Differences in gray matter volume corresponding to delusion and hallucination in patients with schizophrenia compared with patients who have bipolar disorder
}

\author{
This article was published in the following Dove Press journal: \\ Neuropsychiatric Disease and Treatment \\ 18 May 2015 \\ Number of times this article has been viewed
}

\author{
Jinuk Song' \\ Doug Hyun Han' \\ Sun Mi Kim' \\ Ji Sun Hong' \\ Kyung Joon Min' \\ Jae Hoon Cheong ${ }^{2}$ \\ Bung Nyun Kim ${ }^{3}$ \\ 'Department of Psychiatry, Chung \\ Ang University Hospital, ${ }^{2}$ Uimyung \\ Research Institute for Neuroscience, \\ Samyook University, ${ }^{3}$ Department \\ of Psychiatry, Seoul National Hospital, \\ Seoul, South Korea
}

Correspondence: Doug Hyun Han Department of Psychiatry, Chung Ang University Hospital, Heukseok Ro I02, Seoul, 156-755, South Korea

$\mathrm{Tel}+82262993132$

Fax +82 262981508

Email hduk@yahoo.com
Background: Although schizophrenia and bipolar disorder (BD) are classified as different disease entities, they share critical pathognomonic symptoms in terms of hallucination and delusion. Because the characteristics of clinical symptoms are not sufficient to differentiate schizophrenia from BD, several studies have applied brain imaging methods to provide biological evidence of differences. We compared gray matter (GM) volume differences in schizophrenia and BD patients and examined volumetric differences associated with hallucination and delusion in these two groups.

Methods: Ninety-three schizophrenia patients and $75 \mathrm{BD}$ patients who were followed for at least 3 years in an outpatient department were recruited for this study. Magnetic resonance data from 71 schizophrenia patients and $44 \mathrm{BD}$ patients were obtained using a $3.0 \mathrm{~T}$ scanner. Volumetric differences were analyzed using Matlab 8.0.0 and SPM8 software.

Results: The results showed that delusion symptoms were negatively correlated with GM volume within both frontal and both temporal cortices in the schizophrenia group and were negatively correlated with GM volume within the bilateral frontal cortices in the BD group. Hallucination symptoms were negatively correlated with GM volume within the bilateral frontal, bilateral temporal, and left parietal cortices in the schizophrenia group and were negatively correlated with GM volume within the bilateral frontal, right parietal, occipital, and insular cortices in the BD group.

Conclusion: Delusions in schizophrenia were correlated with GM volume in multiple brain regions, including the frontal, temporal, and parietal cortices, compared to those in patients with BD. Hallucination was associated with temporal lobe GM volume in patients with schizophrenia and with insular cortex GM volume in patients with BD.

Keywords: schizophrenia, bipolar disorder, delusion, hallucination, voxel-based morphometry

\section{Introduction}

\section{Common clinical symptoms in schizophrenia and bipolar disorder}

Although schizophrenia and bipolar disorder(BD) have been classified as different disease entities, they share critical pathognomonic symptoms in terms of hallucination and delusion. ${ }^{1}$ Hallucination is a false sensory perception that occurs in the absence of any relevant external stimulation of the sensory modality involved. Delusion is a false belief based on an incorrect inference about external reality. ${ }^{2}$ Due to different treatment methods and prognosis, early differential diagnosis and determination are 
important for disease management in both schizophrenia and BD. Patients with BD tend to be much more expressive than patients with schizophrenia, with schizophrenia patients being rather monotone in the expression of hallucinations. ${ }^{3}$ Compared with schizophrenia, hallucinations in BD tend to be less severe, more visual, and less often auditory. Hallucinations in BD are most often accompanied by persecutory delusions. ${ }^{4}$ Because the characteristics of clinical symptoms are not sufficient to differentiate schizophrenia from $\mathrm{BD},{ }^{5}$ brain imaging studies have attempted to provide biological evidence of differences and a method of clinical differential diagnosis. ${ }^{6,7}$

\section{Gray matter volume in schizophrenia and bipolar disorder}

Overall, most studies have reported that schizophrenia patients demonstrate gray matter (GM) volume loss in both hemispheres of the brain. During the development of schizophrenia, typically in adolescence, GM volume loss begins to occur, starting in the parietal association cortices and proceeding frontally to envelop the dorsolateral prefrontal cortex and temporal cortices, including the superior temporal gyri, and eventually engulfing parietal, motor and supplementary motor, temporal, and prefrontal cortices. ${ }^{8}$ In comparison with relatives of schizophrenia patients, the most significant losses appear to occur in the left thalamus and right caudate, extending into the cerebrum, parahippocampal gyrus, and hippocampus. ${ }^{9-11}$ Patients with schizophrenia also have a smaller hippocampal volume than healthy controls. GM tends to be reduced in relatives of schizophrenia patients compared with healthy control subjects. ${ }^{9}$ Moreover, the tendency for decreased GM volume within frontal, temporal, and parietal cortices has been observed not only in schizophrenia patients, but also in their unaffected siblings. ${ }^{11}$ Previous studies have reported different GM volume in schizophrenia patients compared with healthy controls, with larger volumes in the temporal and parietal lobes and the anterior cerebellum. ${ }^{10}$ They also tend to show decreased GM volume in prefrontal and temporal regions.

Several reports on BD have suggested reduced GM volume within the frontal, temporal, and basal ganglia regions. GM reduction in the left rostral anterior cingulate cortex and right fronto-insular cortex have been associated with BD., ${ }^{72}$ Fronto-insular cortex abnormalities are not evident in the early phases of the illness. BD patients show cortical thinning, primarily in the frontal lobes and superior temporal and temporoparietal regions. ${ }^{7}$ In chronic BD patients, the longer duration of illness has been associated with increased GM in a cluster that includes the basal ganglia, subgenual anterior cingulate cortex, and amygdala. ${ }^{12}$

Several studies have directly compared GM volume between schizophrenia and BD patients. Compared with schizophrenia patients, BD patients have smaller lateral ventricular volume and greater amygdala volume. ${ }^{6}$ Rimol et $\mathrm{al}^{7}$ conducted a study examining cortical thickness and subcortical volume in schizophrenia and BD groups. While schizophrenia patients showed widespread cortical thinning within the frontal, temporal, occipital, and parietal regions, the BD group showed cortical thinning primarily in the frontal, superior temporal, and temporoparietal regions. ${ }^{7}$ Both groups showed bilateral subcortical volume reduction in the hippocampus, left thalamus, right nucleus accumbens, left cerebellar cortex, and brainstem. ${ }^{7}$

\section{Hypothesis}

Based on existing literature, we hypothesized that the frontal cortex is a common deficit area in both schizophrenia and BD patients with delusion. In particular, we predicted that schizophrenia patients would show greater deficit in regions associated with frontal regions compared with BD subjects, while BD patients with hallucination would show greater deficits in frontal-temporal regions.

\section{Materials and methods Subjects}

We reviewed data from 276 schizophrenia patients and 197 BD patients from 2009 to 2014. Among them, 93 schizophrenia patients and 75 BD patients who had been followed for at least 3 years in the outpatient department were recruited via the Department of Psychiatry at Chung Ang University Hospital. At the time of the magnetic resonance imaging (MRI) scan, all patients were experiencing psychotic symptoms including delusions and hallucinations and had no history of medication in the month prior to the scan. All patients were scanned and then followed for 3 years to ensure the correct diagnosis. A total of eleven schizophrenia patients were excluded due to a concurrent mood episode, and $17 \mathrm{BD}$ patients were excluded because they had no symptoms of delusions or hallucinations. Nine schizophrenia patients and ten BD patients were excluded due to use of medication in the month prior to the MRI scan. Two schizophrenia patients and four BD patients failed MRI scanning due to irritable behavior. Exclusion criteria included: age $\geq 60$ or $\leq 18$ years; contraindication to MRI scanning such as claustrophobia or metal implant; and history of head trauma, a medical disorder, or a neurological disorder. A final total of 71 schizophrenia patients and 44 BD patients were enrolled 
in this study following approval by the Chung Ang University Hospital institutional review board. Written informed consent was provided by all participants. All patients were assessed with the Structured Clinical Interview for DSM-IV Axis I Disorders (SCID-I), Clinician Version, ${ }^{13}$ the Positive and Negative Syndrome Scale, ${ }^{14}$ and the Young Mania Rating Scale (YMRS). ${ }^{15}$ The delusions and hallucinations of all patients were assessed using the subscale scores on the Positive and Negative Syndrome Scale. Handedness of patients was assessed using the Edinburgh Inventory. ${ }^{16}$ All scales were assessed by a trained psychiatrist. Healthy comparison subjects were recruited from the community through advertisements at Chung Ang University Hospital. Age-matched and sex-matched healthy comparison subjects were assessed using the SCID-I, and inclusion criteria were no history of psychiatric disorder, no contraindication for MRI scanning, and no medical/neurological disorders.

\section{Image acquisition}

MRI data from all subjects were obtained at Chung Ang University Hospital using an Intera Achieva 3.0 T scanner (Philips, Amsterdam, the Netherlands). Acquisitions consisted of a set of 180 axial cuts parallel to the anterior commissure-posterior commissure line with a slice thickness of $1 \mathrm{~mm}$ and a voxel size of $1 \times 1 \times 1 \mathrm{~mm}^{3}{ }^{3}$ These measurements were collected using the spoiled gradient recalled acquisition sequence (repetition time $10 \mathrm{msec}$, echo time $4 \mathrm{msec}$, and image matrix 256×256).

\section{Voxel-based morphometry and statistical analysis}

Images were analyzed using Matlab 8.0.0 (Mathworks, Natick, MA, USA) and SPM8 software. Images were reconstructed using the voxel-based morphometry toolbox (http://dbm.neuro.uni-jena.de/vbm/). Each structural image was segmented into GM, white matter, and cerebrospinal fluid after normalization using the template provided by the International Consortium for Brain Mapping template for East Asian Brains. During segmentation, the modulated nonlinear warping only option was used. GM was smoothed with an $8 \mathrm{~mm}$ full-width half maximum Gaussian kernel for subsequent statistical analysis. Voxel-wise comparisons of GM volume were performed between the groups (schizophrenia patients versus BD patients) using analysis of covariance with covariates of age, sex, and duration of medication intake. For assessing correlations between positive symptoms, delusional symptoms, hallucination symptoms, and GM volume across the whole brain, multiple regression analyses were performed, controlling for age, sex, and duration of medication intake. Statistical significance was initially set at uncorrected $P \leq 0.001$. The threshold for statistical significance was then increased to family-wise error (FWE) correction $(P \leq 0.05)$.

Differences in demographic variables were calculated using analysis of variance, the independent $t$-test, and the chi-square test as appropriate. Statistical significance was set at $P \leq 0.05$.

\section{Results \\ Demographic data}

There were no differences in age, sex, or handedness between the three groups. BD patients showed significantly higher YMRS score than schizophrenia patients. Schizophrenia patients showed a higher Positive and Negative Syndrome Scale total score, positive symptoms including delusions and hallucinations, and negative symptoms than BD patients. In addition, schizophrenia patients showed higher rates of persistent delusions and hallucinations compared with BD subjects (Table 1).

\section{Comparison of GM volume between schizophrenia patients, BD patients, and controls}

After controlling for whole GM volume, age, sex, YMRS score, and duration of medication, schizophrenia patients had decreased GM volume in the left frontal precentral gyrus (BA 6/BA 4), left extra nucleus (BA 13), left caudate body, left cerebellum, right frontal precentral gyrus (BA 6), right lentiform nucleus, and right caudate body compared with healthy controls. After controlling for whole GM volume, age, sex, YMRS score, and duration of medication, BD patients showed decreased GM volume in the left middle frontal gyrus, left temporal fusiform gyrus (BA 20), and right parietal postcentral gyrus (BA 43) compared with healthy controls. Neither schizophrenia patients nor BD patients showed increased GM volume in any region compared with healthy comparison subjects (Figure 1; Table 2). Compared with BD patients, schizophrenia patients showed decreased GM volume in the left cerebellum, right temporal lobe, right lentiform nucleus, and putamen (Figure 1; Table 2). Schizophrenia patients did not show increased GM volume in any brain region when compared with $\mathrm{BD}$ patients.

\section{Regression analysis between positive symptoms and brain volume in all patients}

Controlling for age, sex, YMRS score, and duration of medication, there was no significant correlation between positive 
Table I Demographic data

\begin{tabular}{|c|c|c|c|c|}
\hline & $S z(n=7 I)$ & BD $(n=44)$ & $H C(n=35)$ & $F / t / \chi^{2}, P$-value \\
\hline Age (years) & $35.6 \pm 14.7$ & $34.8 \pm \mid 4.1$ & $33.9 \pm 14.5$ & $F=0.16, P=0.85$ \\
\hline Education (years) & $10.8 \pm 1.5$ & $11.5 \pm 2.3$ & $12.4 \pm 1.7$ & $F=1.10, P=0.10$ \\
\hline Sex (male/female) & $29 / 42$ & $19 / 25$ & $\mathrm{II} / 24$ & $\chi^{2}=1.1, P=0.49$ \\
\hline Handedness (R/L) & $68 / 3$ & $42 / 2$ & $33 / 2$ & $\chi^{2}=1.0, P=0.4 \mathrm{I}$ \\
\hline Duration of medication (years) & $7.1 \pm 5.4$ & $7.4 \pm 5.8$ & N/A & $t=0.13, P=0.18$ \\
\hline \multicolumn{5}{|l|}{ Type of medication } \\
\hline Typical APC & 7 & 1 & N/A & \\
\hline Atypical APC & 71 & 43 & N/A & \\
\hline Lithium & 2 & 9 & $\mathrm{~N} / \mathrm{A}$ & \\
\hline Anticonvulsant & 6 & 30 & N/A & \\
\hline \multicolumn{5}{|l|}{ Cumulative dosage (g) } \\
\hline APCs & $\mathrm{I}, 0 \mathrm{II} .4 \pm \mathrm{I} 37.5$ & $204.1 \pm 16.9$ & $N / A$ & \\
\hline Lithium & $220.5 \pm 37.4$ & $489.2 \pm 37.6$ & N/A & \\
\hline Anticonvulsant & $323.3 \pm 32.5$ & $751.5 \pm 73.8$ & $\mathrm{~N} / \mathrm{A}$ & \\
\hline YMRS & $14.1 \pm 7.9$ & $23.9 \pm 12.2$ & $\mathrm{~N} / \mathrm{A}$ & $t=4.74, P<0.0 I^{*}$ \\
\hline PANSS total & $80.3 \pm 12.2$ & $70.3 \pm 9.1$ & $\mathrm{~N} / \mathrm{A}$ & $t=4.99, P<0.01 *$ \\
\hline Positive symptoms & $25.5 \pm 5.3$ & $22.3 \pm 5.4$ & $\mathrm{~N} / \mathrm{A}$ & $t=3.1 \mathrm{I}, P<0.01 *$ \\
\hline Delusion & $5.1 \pm 1.1$ & $3.0 \pm 1.8$ & $N / A$ & $t=7.82, P<0.01 *$ \\
\hline Persistent delusion & 17 (23.9\%) & $5(9.1 \%)$ & $\mathrm{N} / \mathrm{A}$ & $\chi^{2}=4.0, P=0.04 *$ \\
\hline Hallucination & $3.9 \pm 2.0$ & $2.3 \pm 1.8$ & $N / A$ & $t=4.4 \mathrm{I}, P<0.0 \mathrm{I} *$ \\
\hline Persistent hallucination & II (I5.5\%) & I (2.2\%) & $\mathrm{N} / \mathrm{A}$ & $\chi^{2}=5.1, P=0.02 *$ \\
\hline Negative symptoms & $15.2 \pm 5.1$ & $10.8 \pm 2.6$ & $\mathrm{~N} / \mathrm{A}$ & $t=5.26, P<0.0 I^{*}$ \\
\hline \multicolumn{5}{|l|}{ Number of patients } \\
\hline Only delusion & 17 & 10 & $N / A$ & \\
\hline Only hallucination & 1 & 1 & N/A & \\
\hline Both & 52 & 18 & N/A & \\
\hline
\end{tabular}

Note: * Statistically significant.

Abbreviations: Sz, schizophrenia; BD, bipolar disorder; HC, healthy control; APC, antipsychotics; YMRS, Young Mania Rating Scale; PANSS, Positive and Negative Syndrome Scale; R/L, right/left.

symptom score and GM volume across all patients. Controlling for age, sex, YMRS score, and duration of medication, delusion score was negatively correlated with GM volume in the left hippocampus across all patients $(\mathrm{x}, \mathrm{y}, \mathrm{z},-32,-43,4$, $\left.r=-0.35, \kappa_{\mathrm{e}}=24, T=3.35, P_{\text {uncorr }}<0.0001\right)$. Controlling for age, sex, YMRS score, and duration of medication, hallucination score was negatively correlated with GM volume in the right uncus across all patients (x, y, z, 12, 0, -30, $r=-0.34, \kappa_{\mathrm{e}}=26$, $\left.T=3.07, P_{\text {uncorr }}<0.0001\right)$.

\section{Regression analysis between positive symptoms and GM volume in schizophrenia}

Controlling for age, sex, YMRS score, and duration of medication, positive symptom score was negatively correlated with GM volume in the left superior frontal gyrus (BA 6, $\left.r=-0.37, \kappa_{\mathrm{e}}=54, P_{\text {uncorr }}<0.0001\right)$, left medial frontal gyrus (BA 6, $r=-0.45, \kappa_{\mathrm{e}}=143, P_{\text {uncorr }}<0.0001$ ), left middle temporal gyrus (BA 21, $r=-0.47, \kappa_{\mathrm{e}}=57, P_{\text {uncorr }}<0.0001$ ), left inferior temporal gyrus (BA 20, $r=-0.41, \kappa_{\mathrm{e}}=78$, $P_{\text {uncorr }}<0.0001$ ), and left fusiform gyrus (BA 37, $r=-0.52$, $\kappa_{\mathrm{e}}=126, P_{\text {uncorr }}<0.0001$ ) in schizophrenia patients (Table 3; Figure 2). Controlling for age, sex, YMRS score, and duration of medication, delusion score was negatively correlated with GM volume in the left superior parietal gyrus (BA 7, $\left.r=-0.47, \kappa_{\mathrm{e}}=159, P_{\text {uncorr }}<0.0001\right)$, left middle temporal gyrus (BA 22, $r=-0.45, \kappa_{\mathrm{e}}=361, P_{\text {uncorr }}<0.0001$ ), left inferior temporal gyrus (BA 20, $r=-0.46, \kappa_{\mathrm{e}}=201, P_{\text {uncorr }}<0.0001$ ), and right parahippocampal gyrus (BA 27, $r=-0.41, P<0.01$ ) in schizophrenia patients (Table 3; Figure 2). Controlling for age, sex, YMRS score, and duration of medication, hallucination score was negatively correlated with GM volume in the left superior frontal gyrus (BA 6, $r=-0.67, \kappa_{\mathrm{e}}=656$, FWE $<0.05, P=0.04$ ), left middle frontal gyrus (BA 47, $\left.r=-0.46, \kappa_{\mathrm{e}}=470, P_{\text {uncorr }}<0.0001\right)$, left inferior parietal lobe (BA 40, $r=-0.45, \kappa_{\mathrm{e}}=234, P_{\text {uncorr }}<0.0001$ ), left middle temporal gyrus (BA 21, $r=-0.68, \kappa_{\mathrm{e}}=1,354$, FWE $<0.05$, $P<0.01$ ), right superior frontal gyrus (BA 9, $r=-0.44$, $\left.\kappa_{\mathrm{e}}=484, P_{\text {uncorr }}<0.0001\right)$, right temporal supramarginal gyrus (BA 40, $r=-0.67, \kappa_{\mathrm{e}}=931, \mathrm{FWE}<0.05, P=0.02$ ), and right inferior temporal gyrus (BA 20, $r=-0.44, \kappa_{\mathrm{e}}=228, P_{\text {uncorr }}<0.0001$ ) in schizophrenia patients (Table 3; Figure 2). 


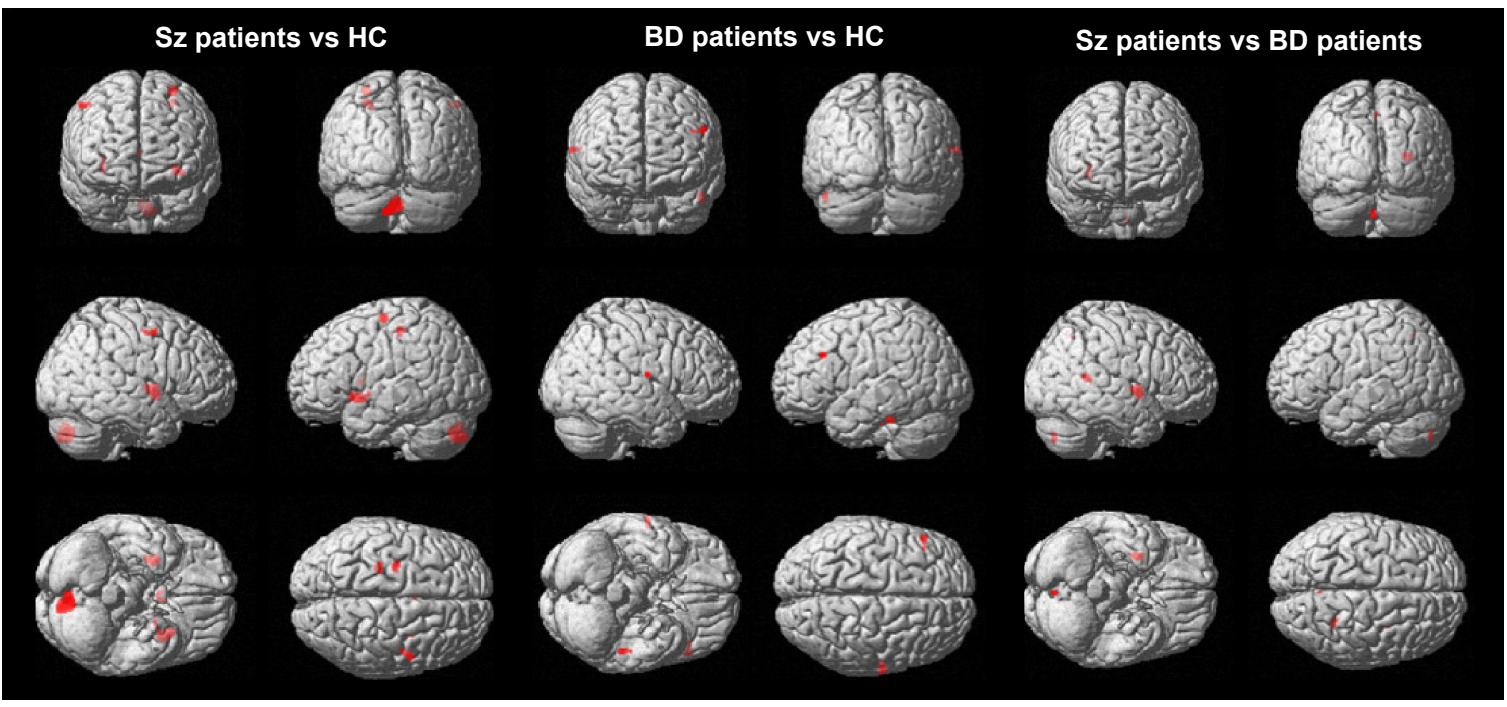

Figure I Regions with significant gray matter differences in group comparisons. Abbreviations: Sz, schizophrenia; BD, bipolar disorder; HC, healthy control.

\section{Regression analysis between positive symptoms and GM volume in BD}

Controlling for whole brain GM volume, age, sex, YMRS score, and duration of medication, positive symptom score was negatively correlated with GM in the left inferior frontal gyrus (BA 47, $r=-0.62, \kappa_{\mathrm{e}}=126, \mathrm{FWE}<0.05$, $P<0.01$ ), left parietal postcentral gyrus (BA 2, $r=-0.69$, $\kappa_{\mathrm{e}}=143$, FWE $<0.05, P<0.01$ ), left inferior parietal lobe (BA $\left.40, r=-0.51, \kappa_{\mathrm{e}}=47, \mathrm{FWE}<0.05, P<0.01\right)$, left superior temporal gyrus $\left(\mathrm{BA} 22, r=-0.69, \kappa_{\mathrm{e}}=479, \mathrm{FWE}<0.05, P<0.01\right.$ ), and right superior frontal gyrus $\left(\mathrm{BA} 9, r=-0.50, \kappa_{\mathrm{e}}=44, \mathrm{FWE}\right.$ $<0.05, P<0.01$ ) in BD patients (Table 4; Figure 2). Controlling for age, sex, YMRS score, and duration of medication, delusion score was negatively correlated with GM volume in the left middle frontal gyrus (BA 10, $r=-0.45, \kappa_{\mathrm{e}}=113$, $P_{\text {uncorr }}<0.0001$ ), left inferior frontal gyrus (BA 44, $r=-0.47$, $\left.\kappa_{\mathrm{e}}=293, P_{\text {uncorr }}<0.0001\right)$, and right superior frontal gyrus $\left(\mathrm{BA} 9, r=-0.49, \kappa_{\mathrm{e}}=340, P_{\text {uncorr }}<0.0001\right)$ in BD patients (Table4; Figure 2). Controlling for age, sex, YMRS score, and duration of medication, hallucination score was negatively correlated

Table 2 Regions with significant gray matter differences in group comparisons

\begin{tabular}{|c|c|c|c|c|c|c|}
\hline \multicolumn{3}{|c|}{ Talairach coordinates } & \multirow{2}{*}{$\kappa_{\mathrm{e}}$} & \multirow[t]{2}{*}{$T$} & \multirow[t]{2}{*}{$P$-value } & \multirow[t]{2}{*}{ Regions } \\
\hline $\mathbf{x}$ & $y$ & $\mathbf{z}$ & & & & \\
\hline \multicolumn{7}{|c|}{ Sz patients $<\mathrm{HC}$ subjects } \\
\hline-29 & -13 & 63 & 85 & 3.44 & $P_{\text {uncorr }}<0.0001$ & Left frontal precentral gyrus, BA 6 \\
\hline-27 & -28 & 52 & 78 & 3.39 & $P_{\text {uncorr }}<0.0001$ & Left frontal precentral gyrus, BA 4 \\
\hline-33 & II & -8 & 269 & 3.46 & $P_{\text {uncorr }}<0.0001$ & Left extra-nuclear, BA I3 \\
\hline-3 & 6 & 7 & 48 & 3.35 & $P_{\text {uncorr }}<0.0001$ & Left caudate body \\
\hline-2 & -76 & -41 & 500 & 3.66 & $P_{\text {uncorr }}<0.0001$ & Left cerebellum, inferior semi-lunar lobule \\
\hline 53 & 0 & 51 & 59 & 3.32 & $P_{\text {uncorr }}<0.0001$ & Right frontal precentral gyrus, BA 6 \\
\hline 30 & -1 & 1 & 254 & 3.62 & $P_{\text {uncorr }}<0.0001$ & Right lentiform nucleus, putamen \\
\hline 2 & 3 & 9 & 42 & 3.25 & $P_{\text {uncorr }}<0.0001$ & Right caudate body \\
\hline \multicolumn{7}{|c|}{ BD patients $<\mathrm{HC}$ subjects } \\
\hline-51 & 27 & 31 & 48 & 3.61 & $P_{\text {uncorr }}<0.0001$ & Left middle frontal gyrus, BA 9 \\
\hline-50 & -31 & -29 & 60 & 3.88 & $P_{\text {uncorr }}<0.0001$ & Left temporal fusiform gyrus, BA 20 \\
\hline 63 & -12 & 15 & 43 & 3.95 & $P_{\text {uncorr }}<0.0001$ & Right parietal postcentral gyrus, BA 43 \\
\hline \multicolumn{7}{|c|}{ Sz patients $<$ BD patients } \\
\hline-4 & -76 & -44 & 48 & 3.58 & $P_{\text {uncorr }}<0.001$ & Left cerebellum, inferior semi-lunar lobule \\
\hline 29 & -46 & 13 & 72 & 3.74 & $P_{\text {uncorr }}<0.001$ & Right temporal lobe \\
\hline 32 & -1 & I & 172 & 3.90 & $P_{\text {uncorr }}<0.001$ & Right lentiform nucleus, putamen \\
\hline
\end{tabular}

Note: $P_{\text {uncorr }}$ indicates the uncorrected $P$-value.

Abbreviations: $\mathrm{Sz}$, schizophrenia; BD, bipolar disorder; $\kappa_{\mathrm{e}}$, voxel size; BA, Brodmann's area; HC, healthy control. 
Table 3 Correlations between positive symptoms and gray matter volume in patients with schizophrenia

\begin{tabular}{|c|c|c|c|c|c|c|}
\hline \multicolumn{3}{|c|}{ Talairach coordinates } & \multirow[t]{2}{*}{$\kappa_{e}$} & \multirow[t]{2}{*}{$T$} & \multirow[t]{2}{*}{$P$-value } & \multirow[t]{2}{*}{ Regions } \\
\hline $\mathbf{x}$ & y & $\mathbf{z}$ & & & & \\
\hline \multicolumn{7}{|c|}{ Positive symptoms in schizophrenia } \\
\hline-2 & 3 & 64 & 54 & 3.69 & $P_{\text {uncorr }}<0.0001$ & Left superior frontal gyrus, BA 6 \\
\hline-14 & -7 & 58 & 143 & 3.77 & $P_{\text {uncorr }}<0.0001$ & Left medial frontal gyrus, BA 6 \\
\hline-3 & 29 & 58 & 57 & 3.76 & $P_{\text {uncorr }}<0.0001$ & Left medial frontal gyrus, BA 6 \\
\hline-68 & -12 & -14 & 58 & 3.62 & $P_{\text {uncorr }}<0.0001$ & Left middle temporal gyrus, BA 2 I \\
\hline-47 & 2 & -20 & 56 & 3.80 & $P_{\text {uncorr }}<0.0001$ & Left middle temporal gyrus, BA 2I \\
\hline-63 & -37 & -18 & 78 & 3.78 & $P_{\text {uncorr }}<0.0001$ & Left inferior temporal gyrus, BA 20 \\
\hline-56 & -52 & -14 & 126 & 3.96 & $P_{\text {uncorr }}<0.0001$ & Left temporal fusiform gyrus, BA 37 \\
\hline \multicolumn{7}{|c|}{ Delusion in schizophrenia } \\
\hline-35 & -63 & 54 & 159 & 3.98 & $P_{\text {uncorr }}<0.0001$ & Left superior parietal lobule, BA 7 \\
\hline-66 & -13 & -15 & 119 & 4.06 & $P_{\text {uncorr }}<0.0001$ & Left middle temporal gyrus, BA 2I \\
\hline-56 & -49 & 3 & 361 & 3.97 & $P_{\text {uncorr }}<0.0001$ & Left middle temporal gyrus, BA 22 \\
\hline-65 & -39 & -18 & 201 & 4.02 & $P_{\text {uncorr }}^{\text {uncorr }}<0.0001$ & Left inferior temporal gyrus, BA 20 \\
\hline 24 & -33 & -2 & 106 & 3.78 & $P_{\text {uncorr }}<0.0001$ & Right parahippocampal gyrus, BA 27 \\
\hline \multicolumn{7}{|c|}{ Hallucination in schizophrenia } \\
\hline-14 & 3 & 70 & 656 & 4.31 & FWE $<0.05, P=0.04$ & Left superior frontal gyrus, BA 6 \\
\hline-47 & 48 & -11 & 470 & 4.14 & $P_{\text {uncorr }}<0.0001$ & Left middle frontal gyrus, BA 47 \\
\hline-38 & -36 & 46 & 234 & 3.90 & $P_{\text {uncorr }}<0.0001$ & Left inferior parietal lobule, BA 40 \\
\hline-56 & -54 & 13 & 757 & 4.76 & FWE $<0.05, P=0.03$ & Left middle temporal gyrus, BA 39 \\
\hline-60 & -19 & -11 & $\mathrm{I}, 354$ & 4.54 & $\mathrm{FWE}<0.05, P<0.01$ & Left middle temporal gyrus, BA 2I \\
\hline 17 & 53 & 34 & 484 & 3.94 & $P_{\text {uncorr }}<0.0001$ & Right superior frontal gyrus, BA 9 \\
\hline 26 & 59 & 7 & 174 & 3.77 & $P_{\text {uncorr }}^{\text {uncorr }}<0.0001$ & Right superior frontal gyrus, BA 10 \\
\hline 57 & -48 & 19 & 931 & 4.38 & $\begin{array}{l}\text { uncorr } \\
\text { FWE }<0.05, P=0.02\end{array}$ & Right temporal supramarginal gyrus, BA 40 \\
\hline 65 & -25 & -20 & 228 & 3.98 & $P_{\text {uncorr }}<0.0001$ & Right inferior temporal gyrus, BA 20 \\
\hline
\end{tabular}

Note: $P_{\text {uncorr }}$ indicates the uncorrected $P$-value.

Abbreviations: FWE, family-wise error; $\kappa_{\mathrm{e}}$, voxel size; BA, Brodmann's area.
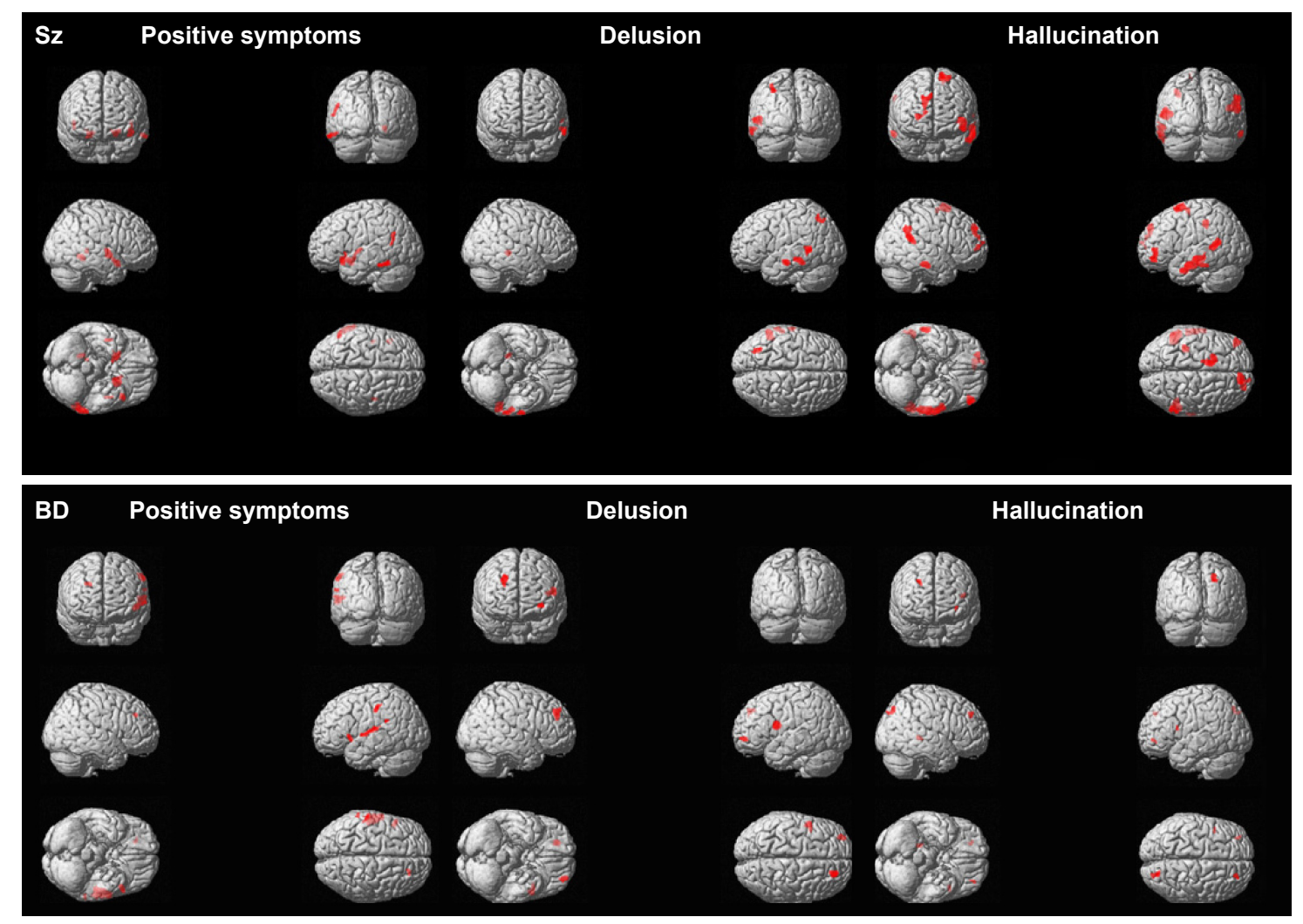

Figure 2 Correlations between symptoms and gray matter volume in schizophrenia patients and bipolar disorder patients. Abbreviations: Sz, schizophrenia; BD, bipolar disorder. 
Table 4 Correlations between positive symptoms and gray matter volume in patients with bipolar disorder

\begin{tabular}{|c|c|c|c|c|c|c|}
\hline \multicolumn{3}{|c|}{ Talairach code } & \multirow[t]{2}{*}{$\kappa_{e}$} & \multirow[t]{2}{*}{$\boldsymbol{F}$} & \multirow[t]{2}{*}{$P$-value } & \multirow[t]{2}{*}{ Regions } \\
\hline $\mathbf{x}$ & $\mathbf{y}$ & $\mathbf{z}$ & & & & \\
\hline \multicolumn{7}{|c|}{ Positive symptoms in BD } \\
\hline-50 & 17 & -2 & 126 & 5.90 & FWE $<0.05, P<0.01$ & Left inferior frontal gyrus, BA 47 \\
\hline-59 & -27 & 43 & 143 & 6.19 & FWE $<0.05, P<0.01$ & Left parietal postcentral gyrus, BA 2 \\
\hline-62 & -40 & 24 & 47 & 5.65 & FWE $<0.05, P<0.0$ I & Left inferior parietal, BA 40 \\
\hline-60 & -4 & 3 & 479 & 6.00 & FWE $<0.05, P<0.0$ I & Left superior temporal gyrus, BA 22 \\
\hline 21 & 38 & 31 & 44 & 5.56 & FWE $<0.05, P<0.0$ I & Right superior frontal gyrus, BA 9 \\
\hline \multicolumn{7}{|c|}{ Delusion in BD } \\
\hline-32 & 53 & -3 & 113 & 3.81 & $P_{\text {uncorr }}<0.0001$ & Left middle frontal gyrus, BA 10 \\
\hline-51 & 5 & 18 & 293 & 3.88 & $P_{\text {uncorr }}<0.0001$ & Left inferior frontal gyrus, BA 44 \\
\hline 23 & 42 & 33 & 340 & 3.98 & $P_{\text {uncorr }}<0.0001$ & Right superior frontal gyrus, BA 9 \\
\hline \multicolumn{7}{|c|}{ Hallucination in BD } \\
\hline-33 & 50 & -2 & 47 & 3.24 & $P_{\text {uncorr }}<0.0001$ & Left middle frontal gyrus, BA 10 \\
\hline-42 & II & 15 & 68 & 3.27 & $P_{\text {uncorr }}<0.0001$ & Left insula, BA I 3 \\
\hline 26 & 45 & 36 & 62 & 3.21 & $P_{\text {uncorr }}<0.0001$ & Right superior frontal gyrus, BA 9 \\
\hline 21 & -76 & 43 & 159 & 3.59 & $P_{\text {uncorr }}<0.0001$ & Right parietal precuneus, BA 7 \\
\hline 20 & -39 & I & 69 & 3.25 & $P_{\text {uncorr }}<0.0001$ & Right occipital lingual gyrus, BA 30 \\
\hline
\end{tabular}

Note: $P_{\text {uncorr }}$ indicates the uncorrected $P$-value.

Abbreviations: BD, bipolar disorder; FWE, family-wise error; $\kappa_{\mathrm{e}}$, voxel size; BA, Brodmann's area.

with GM volume in the left middle frontal gyrus (BA 10, $r=-0.37, \kappa_{\mathrm{e}}=47, P_{\text {uncorr }}<0.0001$ ), left insula (BA 13, $r=-0.37$, $\kappa_{\mathrm{e}}=68, P_{\text {uncorr }}<0.0001$ ), right superior frontal gyrus (BA 9, $\left.r=-0.36, \kappa_{\mathrm{e}}=62, P_{\text {uncorr }}<0.0001\right)$, right parietal precuneus (BA 7, $\left.r=-0.41, \kappa_{\mathrm{e}}=159, P_{\text {uncorr }}<0.0001\right)$, and right occipital lingual gyrus (BA 30, $\left.r=-0.37, \kappa_{\mathrm{e}}=69, P_{\text {uncorr }}<0.0001\right)$ in BD patients (Table 4; Figure 2).

\section{Discussion}

Compared with previous studies, the goal in this study was focused on differences in GM volume between schizophrenia patients and BD patients corresponding to psychiatric symptoms of delusions and hallucinations. Our results showed that delusion symptoms were negatively correlated with GM volume within the bilateral frontal and bilateral temporal cortices in schizophrenia patients and were negatively correlated with GM volume within the bilateral frontal cortices in BD patients. We also found that hallucination symptoms were negatively correlated with GM volume in the bilateral frontal, bilateral temporal, and left parietal cortices in schizophrenia patients and were negatively correlated with GM volume in the bilateral frontal, right parietal, and occipital cortices in BD patients.

\section{Comparison of GM volume between schizophrenia and BD patients}

In a direct comparison of GM volume between schizophrenia and BD patients, we found that schizophrenia patients showed decreased GM volume within the putamen, temporal lobe, and cerebellum when compared with BD patients. These results differ somewhat from previous studies suggesting that schizophrenia patients show widespread thinning of the cortex, including the frontal, temporal, parietal, and occipital cortices, when compared with BD patients, who tend to show localized thinning in small regions within the frontal and temporal cortices. ${ }^{6,7}$ In addition, subcortical volume structures, including the hippocampus, thalamus, and nucleus accumbens, as well as the left cerebellar cortex, tend to be reduced in both schizophrenia and BD patients. ${ }^{?}$

\section{Correlation between delusions and GM volume in schizophrenia and BD patients}

In schizophrenia patients, we found that delusions were negatively correlated with GM volume in the parietal, frontal, and temporal lobes. However, in BD patients, delusions were correlated only with the frontal lobe. Because dopamine regulation occurs in the frontal and striatal areas, the frontal lobe is well known to be associated with delusions in patients with schizophrenia. ${ }^{17,18}$ In addition to the frontal lobe, other brain areas including the temporal and parietal cortices are also associated with production of delusions in patients with schizophrenia. Rimol et $\mathrm{al}^{7}$ reported that schizophrenia patients showed reduced GM volume within the temporal and subcortical areas, including the hippocampus, left thalamus, right nucleus accumbens, cerebellum, and brainstem, compared with control subjects. In a 5-year follow-up MRI study, van Haren et $\mathrm{al}^{19}$ reported extensive loss of GM volume within the left superior frontal area, left superior temporal gyrus, 
right caudate nucleus, and right thalamus in patients with schizophrenia. In a 4-year follow-up MRI study, Mathalon et $\mathrm{al}^{20}$ also reported GM volume decline within the right frontal and bilateral posterior superior temporal regions in schizophrenia patients compared with healthy comparison subjects. Moreover, Takahashi et $\mathrm{al}^{21}$ reported that GM volume reduction within the superior temporal gyrus is correlated with severity of delusions in patients with schizophrenia. Hypoactivity of the frontotemporal area in response to reality monitoring paradigms was associated with the severity of delusion in patients with schizophrenia. ${ }^{22}$ The contribution of the frontal lobe and other areas, including the temporal and parietal areas, to the production of delusions in schizophrenia can be explained with the salience attribution hypothesis. ${ }^{23}$ Phasic firing of dopaminergic neurons projecting to the striatum in schizophrenia may attribute incentive salience to irrelevant stimuli and thus contribute to delusional moods and delusion formation. ${ }^{24}$ Pankow et al suggested that incentive salience is seen not only in the frontostriatal area, but also in limbic regions such as the amygdala during interaction with prefrontal and temporal cortices and may be associated with formation of delusions and negative symptoms. ${ }^{25}$ Based on these previous findings, we cautiously suggest that delusions in schizophrenia may be associated with deficits in multiple regions compared with those in $\mathrm{BD}$.

\section{Correlation between hallucinations and GM volume in schizophrenia and $\mathrm{BD}$ patients}

In patients with schizophrenia, hallucination was negatively correlated with GM volume in the frontal, temporal, and parietal lobes. Hallucination in patients with BD was negatively correlated with GM in the insular cortex and in the frontal, parietal, and occipital lobes. Many studies have suggested that the reduced volume in the frontal and temporal cortices is associated with auditory hallucinations in patients with schizophrenia and in patients with psychosis. Gaser et $\mathrm{al}^{26}$ reported that auditory hallucinations are associated with reduced volume within the transverse temporal gyrus and middle and inferior frontal gyri. In a quantitative meta-analysis of voxel morphometry studies, Modinos et $\mathrm{al}^{27}$ suggested that reduced volume of GM within the superior temporal cortex is positively correlated with auditory hallucinations in schizophrenia. The insular cortex, a region of the paralimbic system, is known to have an important role in sensory integration, auditory hallucination, and language. ${ }^{28}$ In a review of cortical or subcortical GM abnormalities in first-degree relatives of BD patients, Nery et $\mathrm{al}^{29}$ suggested that the insular cortex is a structural vulnerability factor of BD. However, Stanfield et $\mathrm{al}^{30}$ reported that the reduction in volume within the left middle temporal gyrus is associated with hallucination score in BD patients. Future studies should focus on brain areas and hallucination symptoms in $\mathrm{BD}$ patients.

\section{Limitations}

There were several limitations to the current study. First, although we recruited patients who had not used medication within 1 month prior to the MRI scan and showed no differences in drug history, medication effects cannot be ruled out. Second, because this study focused on neuroimaging evaluation, other neuropsychological variables, such as intelligence, attention, and educational level, were not assessed in this study. Such neuropsychological variables may have affected the study findings. Third, because the study was cross-sectional, we could fully investigate the relationships between brain regions and psychotic symptoms. Therefore, caution is needed when interpreting the correlations between GM volume and delusion and hallucination scores. In the current study and previous studies, ${ }^{21,26,27}$ there were significant differences in the persistence of delusions and hallucinations between schizophrenia and BD patients. Future studies should compare longitudinal changes in GM corresponding to delusions and hallucinations between schizophrenia and BD patients.

\section{Conclusion}

Delusions in schizophrenia patients were negatively correlated with GM across multiple brain regions, including the frontal, temporal, and parietal cortices. Hallucination in patients with schizophrenia was associated with the temporal lobe, while hallucination in patients with $\mathrm{BD}$ was associated with the insular cortex.

\section{Acknowledgment}

This work was supported by the Korean Health Technology R\&D Project, Ministry of Health and Welfare, Republic of Korea (A120013).

\section{Disclosure}

The authors report no conflicts of interest in this work.

\section{References}

1. Altamura AC, Buoli M, Pozzoli S. Role of immunological factors in the pathophysiology and diagnosis of bipolar disorder: comparison with schizophrenia. Psychiatry Clin Neurosci. 2014;68(1):21-36.

2. Sadock BJ, Sadock VA, Ruiz P. Kaplan and Sadock's Synopsis of Psychiatry: Behavioral Sciences/Clinical Psychiatry. 11th ed. Philadelphia, PA, USA: Wolters Kluwer; 2014. 
3. Myers N, Bhatty S, Broussard B, Compton M. Clinical correlates of initial treatment disengagement in first-episode psychosis. Clin Schizophr Relat Psychoses. November 3, 2014. [Epub ahead of print].

4. Baethge C, Baldessarini RJ, Freudenthal K, Streeruwitz A, Bauer M, Bschor T. Hallucinations in bipolar disorder: characteristics and comparison to unipolar depression and schizophrenia. Bipolar Disord. 2005; 7(2):136-145.

5. Maier W, Zobel A, Wagner M. Schizophrenia and bipolar disorder: differences and overlaps. Curr Opin Psychiatry. 2006;19(2):165-170.

6. Arnone D, Cavanagh J, Gerber D, Lawrie SM, Ebmeier KP, McIntosh AM. Magnetic resonance imaging studies in bipolar disorder and schizophrenia: meta-analysis. Br J Psychiatry. 2009;195(3):194-201.

7. Rimol LM, Hartberg CB, Nesvag R, et al. Cortical thickness and subcortical volumes in schizophrenia and bipolar disorder. Biol Psychiatry. 2010;68(1):41-50.

8. Thompson PM, Vidal C, Giedd JN, et al. Mapping adolescent brain change reveals dynamic wave of accelerated gray matter loss in very early-onset schizophrenia. Proc Natl Aacd Sci U S A. 2001;98(20): 11650-11655.

9. Boos HB, Aleman A, Cahn W, Hulshoff Pol H, Kahn RS. Brain volumes in relatives of patients with schizophrenia: a meta-analysis. Arch Gen Psychiatry. 2007;64(3):297-304.

10. Watson DR, Anderson JM, Bai F, et al. A voxel based morphometry study investigating brain structural changes in first episode psychosis. Behav Brain Res. 2012;227(1):91-99.

11. Honea RA, Meyer-Lindenberg A, Hobbs KB, et al. Is gray matter volume an intermediate phenotype for schizophrenia? A voxel-based morphometry study of patients with schizophrenia and their healthy siblings. Biol Psychiatry. 2008;63(5):465-474.

12. Bora E, Fornito A, Yucel M, Pantelis C. Voxelwise meta-analysis of gray matter abnormalities in bipolar disorder. Biol Psychiatry. 2010; 67(11):1097-1105.

13. First MB, Spitzer RL, Gibbon M, Williams JB. Structured Clinical Interview for DSM-IV Axis I Disorders (SCID-I), Clinician Version. Arlington, VA, USA: American Psychiatric Association; 2012.

14. Kay SR, Fiszbein A, Opler LA. The Positive and Negative Syndrome Scale (PANSS) for schizophrenia. Schizophr Bull. 1987;13(2): 261-276.

15. Young RC, Biggs JT, Ziegler VE, Meyer DA. A rating scale for mania: reliability, validity and sensitivity. Br J Psychiatry. 1978;133: 429-435.

16. Oldfield RC. The assessment and analysis of handedness: the Edinburgh Inventory. Neuropsychologia. 1971;9(1):97-113.

17. Abercrombie ED, Keefe KA, DiFrischia DS, Zigmond MJ. Differential effect of stress on in vivo dopamine release in striatum, nucleus accumbens, and medial frontal cortex. J Neurochem. 1989;52(5): 1655-1658.
18. Abi-Dargham A, Mawlawi O, Lombardo I, et al. Prefrontal dopamine D1 receptors and working memory in schizophrenia. JNeurosci. 2002; 22(9):3708-3719.

19. van Haren NE, Hulshoff Pol HE, Schnack HG, et al. Focal gray matter changes in schizophrenia across the course of the illness: a 5-year follow-up study. Neuropsychopharmacology. 2007;32(10):2057-2066.

20. Mathalon DH, Sullivan EV, Lim KO, Pfefferbaum A. Progressive brain volume changes and the clinical course of schizophrenia in men: a longitudinal magnetic resonance imaging study. Arch Gen Psychiatry. 2001;58(2):148-157.

21. Takahashi T, Wood SJ, Yung AR, et al. Progressive gray matter reduction of the superior temporal gyrus during transition to psychosis. Arch Gen Psychiatry. 2009;66(4):366-376.

22. Thoresen C, Endestad T, Sigvartsen NP, et al. Frontotemporal hypoactivity during a reality monitoring paradigm is associated with delusions in patients with schizophrenia spectrum disorders. Cogn Neuropsychiatry. 2014;19(2):97-115.

23. Heinz A, Schlagenhauf F. Dopaminergic dysfunction in schizophrenia salience attribution revisited. Schizophr Bull. 2010;36(3):472-485.

24. Robinson TE, Berridge $\mathrm{KC}$. The neural basis of drug craving: an incentive-sensitization theory of addiction. Brain Res Brain Res Rev. 1993;18(3):247-291.

25. Pankow A, Knobel A, Voss M, Heinz A. Neurobiological correlates of delusion: beyond the salience attribution hypothesis Neuropsychobiology. 2012;66(1):33-43.

26. Gaser C, Nenadic I, Volz HP, Buchel C, Sauer H. Neuroanatomy of "hearing voices": a frontotemporal brain structural abnormality associated with auditory hallucinations in schizophrenia. Cerebr Cortex. 2004;14(1):91-96.

27. Modinos G, Costafreda SG, van Tol MJ, McGuire PK, Aleman A, Allen P. Neuroanatomy of auditory verbal hallucinations in schizophrenia: a quantitative meta-analysis of voxel-based morphometry studies. Cortex. 2013;49(4):1046-1055.

28. Takahashi T, Suzuki M, Zhou SY, et al. Volumetric MRI study of the short and long insular cortices in schizophrenia spectrum disorders. Psychiatry Res. 2005;138(3):209-220.

29. Nery FG, Monkul ES, Lafer B. Gray matter abnormalities as brain structural vulnerability factors for bipolar disorder: a review of neuroimaging studies of individuals at high genetic risk for bipolar disorder. Aust N Z J Psychiatry. 2013;47(12):1124-1135.

30. Stanfield AC, Moorhead TW, Job DE, et al. Structural abnormalities of ventrolateral and orbitofrontal cortex in patients with familial bipolar disorder. Bipolar Disord. 2009;11(2):135-144.
Neuropsychiatric Disease and Treatment

\section{Publish your work in this journal}

Neuropsychiatric Disease and Treatment is an international, peerreviewed journal of clinical therapeutics and pharmacology focusing on concise rapid reporting of clinical or pre-clinical studies on a range of neuropsychiatric and neurological disorders. This journa is indexed on PubMed Central, the 'PsycINFO' database and CAS,

\section{Dovepress}

and is the official journal of The International Neuropsychiatric Association (INA). The manuscript management system is completely online and includes a very quick and fair peer-review system, which is all easy to use. Visit http://www.dovepress.com/testimonials.php to read real quotes from published authors. 